\title{
GESTÃO PÚBLICA NA ODONTOLOGIA COM O ADVENTO DO PROGRAMA BRASIL SORRIDENTE.
}

\author{
PUBLIC MANAGEMENT IN DENTISTRY WITH THE ADVENT OF THE
}

SMILLING BRAZIL PROGRAM.

Sérgio Spezzia*

Cirurgião Dentista e Mestre em Ciências pela Escola Paulista de Medicina Universidade Federal de São Paulo.

\section{Autor Principal:}

Sérgio Spezzia

Rua Silva Bueno, 1001

São Paulo - SP

CEP: 04208-050

Tel. (11) 96925.3157

Email: sergio.spezzia@unifesp.br 


\title{
Resumo
}

A instituição no ano de 2004 da nova Política Nacional de Saúde Bucal, intitulada Programa Brasil Sorridente (PBS), trouxe consigo inovação e mudou o quadro odontológico na área de saúde. O objetivo deste artigo foi averiguar a performance da gestão pública odontológica e do PBS no desempenho da assistência à saúde pública odontológica. O PBS trouxe consigo um impacto significativo para alavancar a saúde bucal no Brasil, uma vez que até então não havia pelo governo federal, adoção de medidas satisfatórias para o setor. Os repasses de verba destinados ao custeio da saúde bucal da União para os municípios aumentaram. Constatou-se melhora significativa nos serviços prestados, relacionados a assistência à saúde pública odontológica com a implementação do PBS, comparando-se com a realidade apresentada anteriormente e levando-se em consideração o repasse de verbas escasso de antes.

Palavras-chave: Gestão em Saúde. Sistema Único de Saúde. Iniquidade Social. Saúde Bucal.

\begin{abstract}
Institution in 2004 of the National Oral Health Policy, the Brazilian Smiling Program, brought with it innovation and changed the odontological framework in the health area. The objective of this article was to investigate the performance of public dental management and the Brazilian Smiling Program in the performance of dental public health care. PBS brought with it a significant impact to leverage oral health in Brazil, since until then the federal government had not adopted satisfactory measures for the sector. The onlending of funds destined to the cost of oral health of the Union to the municipalities increased. There was a significant improvement in the services provided, related to dental public health assistance with the implementation of PBS, comparing with the reality presented previously and taking into account the transfer of scarce funds from before.
\end{abstract}

Keywords: Health Management. Unified Health System. Social Inequity. Oral Health. 


\section{INTRODUÇÃO}

A implementação e formulação de políticas é de responsabilidade do poder público, este que deve preocupar-se com o bem estar social do cidadão. Os direitos de cidadania, englobam os direitos ao acesso a saúde bucal, o que deve ocorrer por intermédio da instituição de políticas públicas, que devem ser capazes de assegurar acesso igualitário e de cunho universal as ações e serviços de saúde, parâmetros os quais estão garantidos pela Constituição Cidadã de 1988 (BRASIL, 1988).

Políticas públicas constam de um grupo de medidas executadas pelo poder público, que visam promover a resolução dos problemas apresentados pela coletividade por intermédio de ações que possibilitem a aplicabilidade dos recursos públicos (RUAS, 2012).

Ações de promoção de saúde bucal acham-se inseridas em um contexto amplo de saúde, que integra a saúde bucal as demais práticas de Saúde Coletiva nas diversas áreas da saúde. Na área da saúde bucal, políticas públicas devem ser discutidas continuadamente, visando a obtenção de meios que possibilitem angariar verbas para o setor, verbas estas que até então mostram-se insuficientes. Essa ação tem a finalidade de propiciar equidade e universalidade nos atendimentos prestados à população (SPEZZIA, 2014).

No Brasil como existe grande demanda reprimida nos atendimentos públicos odontológicos de saúde voltados, principalmente para a atenção secundária e terciária, as políticas públicas a serem implementadas podem viabilizar recursos. Nesse contexto, sabe-se que a assistência pública odontológica, fundamentalmente ocorre através da prestação de serviços pela atenção primária com o fornecimento dos serviços básicos, não ocorrendo abrangência satisfatória de atendimentos especializados e tratamentos com maior complexidade. O crescimento da oferta de serviços básicos de saúde bucal promovida pelas instituições públicas não foi acompanhada pelo incremento também de ações de saúde na atenção secundária e terciária, o que demonstra a inexistência de adequados sistemas de referência e contra-referência na saúde bucal (BRASIL, 2004). 
A realidade existente no sistema de saúde brasileiro possui determinantes históricos. O setor saúde foi afligido por influências históricas oriundas de todo o contexto político-social ocorrido em nosso país ao longo dos anos (POLIGNANO, 2005).

A abrangência do atendimento público odontológico por si só, mostrou-se limitada, o que dificultou a elaboração de medidas sólidas, visando amplitude imediata e universal através das políticas públicas, devido ao fato, buscou-se para resolução, instituir políticas compensatórias, firmando metas prioritárias e promovendo $\mathrm{o}$ direcionamento dos recursos públicos com atendimentos seletivos prestados aos indivíduos mais necessitados (OLIVEIRA; SALIBA, 2005). Os alvos prioritários para o direcionamento dos recursos foram crianças e gestantes (CRUZ, 2010).

Os serviços públicos prestados em âmbito bucal, historicamente foram configurados por ações de baixa complexidade, que possuíam acesso restrito e que na maioria das vezes, resultavam em procedimentos curativos e multiladores, com predomínio das exodontias. Os municípios brasileiros procuravam desenvolver ações voltadas prioritariamente para gestantes e indivíduos na faixa de idade escolar básica entre 6 e 12 anos. Adultos e idosos tinham acesso somente aos serviços de pronto atendimento e de urgência, o que designava prática de exclusão social a esse grupo de indivíduos (SPEZZIA et al., 2015).

De acordo com o descrito nas Diretrizes para Atenção em Saúde Bucal da Secretaria Municipal de Saúde do Município de São Paulo - SP em 2009, por anos a assistência à saúde bucal atendia a crianças unicamente com a realização de programas curativos, visando o tratamento da cárie dentária. Medidas preventivas, eventualmente realizadas em escolas realizavam apenas bochechos com solução fluorada (SPEZZIA, 2014).

O setor odontológico permaneceu durante bastante tempo, às margens das políticas públicas de saúde e tinha acesso dificultoso e limitado. Os recursos públicos oferecidos eram escassos e ocorria demora na procura do atendimento odontológico pelos indivíduos, o que repercutia a nível dos procedimentos odontológicos efetuados, fazendo com que as exodontias fossem o principal tratamento preconizado pela rede 
pública, perpetuando a visão da Odontologia mutiladora (BARROS; BERTOLDI, 2002).

Sabe-se que até a instituição no ano de 2004 da nova Política Nacional de Saúde Bucal (PNSB), intitulada Programa Brasil Sorridente (PBS), que trouxe consigo inovação e mudou o quadro odontológico da área de saúde apresentado, não havia política nacional odontológica efetiva, existiam somente algumas experiências isoladas na assistência à saúde bucal que almejavam ampliar o acesso aos serviços, desenvolvendo ações de promoção e prevenção de saúde, e que visavam fornecer atividades curativas com maior complexidade para os atendimentos (NARVAI; FRAZÃO, 2008; MACHADO et al., 2017; MACHADO, 2018).

O objetivo deste artigo foi averiguar a performance da gestão pública odontológica e do Programa Brasil Sorridente no desempenho da assistência à saúde pública odontológica.

\section{REVISÃO DE LITERATURA}

Relacionado a saúde pública odontológica, instituiu-se algumas mudanças, visando aprimoramento assistencial. No ano 2000, incorporou-se a equipe de saúde bucal (ESB) na Estratégia de Saúde da Família (ESF). Outra mudança ocorreu no ano de 2004, já por intermédio do PBS com o estabelecimento dos Centros de Especialidades Odontológicas (CEO). Todas essas mudanças objetivavam ampliar a oferta do atendimento odontológico (NAKAGAWA, 2011). A ESB presente na ESF propiciou a adoção de uma postura mais ativa voltada para a atenção primária e os CEO permitiram oferecimento de tratamentos com maior complexidade, englobando procedimentos radiológicos, protéticos e endodônticos (FRAZÃO; NARVAI, 2009).

A implementação do PBS agiu como um marco voltado para mudança do foco da atenção em saúde bucal, almejando obter melhoria da organização do sistema de saúde como um todo e propondo um modelo que fosse capaz de suprir os princípios básicos preconizados pelo Sistema Único de Saúde (SUS) de equidade, universalidade e integralidade (SCARPARO; ZERMIANI, 2015).

REVISTA FLUMINENSE DE ODONTOLOGIA - ANO XIV - № 50 - Julho / Dezembro 2018 
O PBS visava melhorar a organização do sistema de saúde, procurou-se investir e incrementar a atenção primária, propiciando maiores recursos para o setor, e promovendo atendimento de forma integral, acolhedora e humanizadora. O cirurgião dentista foi inserido no PSF para executar tratamento com cunho preventivo e evitar-se encaminhamento futuro dos pacientes para tratamentos dotados de maior complexidade a nível secundário e terciário, setores onde existe carência de recursos para cobertura. Adotou-se no PBS uma série de medidas com intuito de garantir as ações de promoção, prevenção e recuperação da saúde bucal, visando aprimorar a qualidade de vida da população (BRASIL, 2004).

A PNSB, PBS instituída pretendia promover a reorganização da prática e a qualificação das ações e serviços prestados, agrupando um conjunto de ações voltadas para a saúde bucal que visavam atendimento a indivíduos de todas as idades por intermédio da ampliação do acesso ao tratamento odontológico público e gratuito oferecido pelo SUS. O programa é detentor de algumas linhas de ação principais, tais como: reorganização da atenção básica em saúde bucal, principalmente com a inserção das ESB na ESF e ampliação e qualificação da atenção especializada, especialmente na atenção secundária à saúde com a implementação dos CEO e dos Laboratórios Regionais de Prótese Dentária, contando também com a contribuição da viabilização preventiva de flúor nas águas de abastecimento público. Os CEO constam de estabelecimentos especializados na saúde bucal nos procedimentos da área de diagnóstico bucal com ênfase na averiguação do câncer bucal; periodontia especializada; cirurgia oral menor dos tecidos moles e duros, endodontia e atendimento a pacientes com necessidades especiais (BRASIL, 2004).

Expandiu-se duas intervenções de saúde bucal em nosso país durante as últimas décadas, levando-se em consideração o princípio constitucional da universalização das ações de saúde. Realizou-se a amplificação da fluoretação da água de abastecimento público e do atendimento odontológico na rede pública do SUS. Nesse contexto, as políticas públicas empregadas tiveram cunho essencialmente preventivo e visaram o incremento da atenção primária nas Unidades Básicas de Saúde (UBS), realizando tratamentos preventivos, no intuito de reduzir gastos futuros com a evolução 
desfavorável dos quadros odontológicos, que possivelmente requerirão tratamentos de maior complexidade (BRASIL, 2013).

Os serviços básicos compreendem quase que completamente à disponibilidade de assistência pública odontológica fornecida a população, havendo grande demanda reprimida. No âmbito do SUS, os serviços odontológicos especializados correspondem a aproximadamente $3,5 \%$ dos procedimentos executados, devido ao fato, fica claro que existe baixa capacidade de oferta de serviços odontológicos a serem viabilizados pela atenção secundária e terciária (BRASIL, 2004).

Investiu-se e realizou-se a expansão da atenção básica odontológica e consequentemente ampliou-se a oferta da diversidade de procedimentos no SUS. Fazem-se necessários, porém, investimentos que viabilizem maior acessibilidade do público aos serviços odontológicos nos níveis de atenção secundário e terciário (BRASIL, 2013).

Em termos de Administração Pública em todo mundo, averigua-se a necessidade crescente do ajuste das despesas públicas, visando melhorar a arrecadação de tributos pelo Estado para tornar viável o cumprimento das obrigações com as despesas públicas. A oferta de serviços de saúde encontra-se muitas vezes limitada, devido a falta de recursos financeiros pelo governo e advindo também de barreiras tecnológicas, o que proporciona um desequilíbrio entre oferta e demanda dos serviços.

Por outro lado mesmo com a escassez de recursos, atualmente é possível averiguar que os serviços de saúde, incluindo os serviços odontológicos, almejam realizar o atendimento de maneira integral, acolhedora e humanizada, elementos que são parte integrante do princípio da integralidade dos atendimentos preconizado pelo SUS. O modelo de atenção odontológica vem sofrendo mudanças, assumindo enfoque mais holístico, onde os pacientes passaram a serem vistos como um ser em sua totalidade (SCARPARO; ZERMIANI, 2015; MACHADO et al., 2017).

\section{DISCUSSÃO}

É importante frisar que é necessário instituir políticas públicas efetivas para enfrentamento dos problemas de saúde apresentados no setor odontológico e para 
realizar mudanças. É importante deter conhecimento acerca das condições sociais, devido a desigualdade da distribuição de renda, que estão associadas aos indicadores de atenção básica para tentar-se reduzir as desigualdades também no acesso aos serviços odontológicos, no intuito de promover a equidade nos atendimentos de saúde (ANTUNES; NARVAI, 2010; MAGNO, 2015).

A qualidade da assistência à saúde pública é outro determinante que deve ser avaliado, no que tange a averiguação da existência de uma operacionalidade satisfatória ou não nos atendimentos prestados. Verifica-se, no entanto, que existe muito comumente na prática uma situação de precariedade que se alastra por anos com estruturas físicas impróprias; hospitais públicos com escassez de equipamentos para atendimento; marcação de consultas dificultosa; condições de higiene precárias; falta de insumos básicos, entre outros intempéries (SPEZZIA, 2014; CAPPELLANO, 2018).

Convém salientar que, muitos pacientes mostram-se gratos pelos serviços públicos odontológicos prestados, baseado na dificuldade extremada que é encontrada para os mesmos conseguirem seu atendimento e ainda por temerem que esses serviços que são utilizados deixem de existir. Esse tipo de comportamento mostra-se diretamente intrínsecado a precariedade das condições sócio-econômicas dos pacientes, que se acham impossibilitados de conseguirem para si próprios outra alternativa para atendimento (SPEZZIA, 2014; CAPPELLANO, 2018).

A iniquidade e o agravo das condições sociais dos pacientes são os principais fatores responsáveis pela dificuldade encontrada pelos mesmos quanto a resolução dos seus problemas de saúde. Nesse contexto, as desigualdades sociais impossibilitam o acesso aos serviços odontológicos, devido ao ocorrido políticas públicas devem procurar soluções, visando o desenvolvimento do setor saúde pública (ANTUNES; NARVAI, 2010; MAGNO, 2015).

O serviço público odontológico, além de empreender atendimento clínico, realiza promoção de saúde bucal com atuação junto a comunidade com levantamentos epidemiológicos, ações com cunho preventivo e de educação em saúde (FRAZÃO; NARVAI, 2009).

O PBS trouxe consigo um impacto significativo para alavancar a saúde bucal no Brasil, uma vez que até então não havia pelo governo federal, adoção de medidas 
satisfatórias para o setor. Os repasses de verba destinados ao custeio da saúde bucal da União para os municípios aumentaram (SCARPARO; ZERMIANI, 2015; FRANCO, 2017).

A ampliação do acesso aos serviços de saúde odontológicos ocorreu graças ao PBS, tanto na atenção primária como na atenção secundária. Falta promover a estruturação de uma rede de cuidados para a saúde oral, focada nos princípios básicos do SUS, efetivando a assistência à saúde de forma satisfatória, que seja capaz de englobar todos os níveis de atenção (SPEZZIA, 2014; SPEZZIA et al., 2015).

A redução da desigualdade em saúde por intermédio da qualificação dos serviços odontológicos ofertados, bem como através da organização da prática assistencial foram metas propostas pelo PBS desde sua implantação em 2004 (MACHADO et al., 2017; MACHADO, 2018) .

\section{CONCLUSÃO}

Constatou-se melhora significativa nos serviços prestados, relacionados a assistência à saúde pública odontológica com a implementação do Programa Brasil Sorridente, comparando-se com a realidade apresentada anteriormente e levando-se em consideração o repasse de verbas escasso de antes.

\section{REFERÊNCIAS}

1 - Brasil. Constituição. Constituição: República Federativa do Brasil 1988. Brasília: Senado Federal, Centro Gráfico. 1988, 292p.

2 - Ruas MG. Politicas Públicas. Ministério da Educação. Departamento de Ciências da Administração. UFSC. Florianópolis, 2ª ed, 2012.

3 - Spezzia S. Uma Análise das Políticas Públicas Voltadas para os Serviços de Saúde Bucal no Brasil. [Monografia]. Especialização em Gestão Pública. Pato Branco: Universidade Tecnológica Federal do Paraná, 2014.

4 - Brasil. Ministério da Saúde. Diretrizes da Política Nacional de Saúde Bucal. Secretaria de Atenção à Saúde. Departamento de Atenção Básica. Coordenação Nacional de Saúde Bucal. Brasília, 2004.

REVISTA FLUMINENSE DE ODONTOLOGIA - ANO XIV - No 50 - Julho / Dezembro 2018 
5 - Polignano MV. História das políticas de saúde no Brasil. Uma pequena revisão, 2005. Disponível em: http://www.medicina.ufmg.br/dmps/internato/saude no brasil.rtf Acessado 15 de abril de 2018.

6 - Oliveira JLC, Saliba NA. Atenção odontológica no programa de saúde de Campos dos Goytacazes. Rio de Janeiro, 2005. Disponível em: http://www.scielo.br/scielo.php?script=sci_arttext\&pid=S1413-81232005000500030 Acesso em 15 de fevereiro de 2018.

7 - Cruz DF. A organização do cuidado em saúde bucal de cidades da primeira macroregional de saúde da Paraíba. [Dissertação]. João Pessoa: Universidade Federal da Paraíba, 2010.

8 - Spezzia S, Carvalheiro EM, et al. Uma Análise das Políticas Públicas Voltadas para os Serviços de Saúde Bucal no Brasil. Rev Bras Odontol, 2015; 72(1/2):109-13.

9 - Barros AJD, Bertoldi AD. Desigualdades na utilização e no acesso a serviços odontológicos: uma avaliação em nível nacional. Ciênc Saúde Coletiva, 2002; 7(4):70917.

10 - Narvai PC, Frazão P. Saúde bucal no Brasil: muito além do céu da boca. Rio de Janeiro: Ed Fiocruz, 2008.

11 - Machado CV, Lima LD, et al. Políticas de saúde no Brasil em tempos contraditórios: caminhos e tropeços na construção de um sistema universal. Cad Saude Publica, 2017; 33(Supl. 2):e00129616.

12 - Machado CV. Políticas de Saúde na Argentina, Brasil e México: diferentes caminhos, muitos desafios. Ciênc Saúde Coletiva, 2018; 23(7):2197-212.

13 - Nakagawa MCS. Avaliação das práticas em serviço odontológico na estratégia saúde da família. [Dissertação]. Londrina. Universidade Estadual de Londrina, 2011.

14 - Frazão P, Narvai PC. Saúde bucal no Sistema Único de Saúde: 20 anos de lutas por uma política pública. Saúde em Debate, 2009; 33(81):64-71.

15 - Scarparo A, Zermiani TC, et al. Impacto da Política Nacional de Saúde Bucal Programa Brasil Sorridente - sobre a provisão de serviços odontológicos no Estado do Rio de Janeiro. Cad Saúde Colet, 2015; 23 (4):409-15.

16 - Brasil. Ministério da Saúde. Passo a passo das ações do Brasil Sorridente. Brasília: Ministério da Saúde, 2013.

REVISTA FLUMINENSE DE ODONTOLOGIA - ANO XIV - № 50 - Julho / Dezembro 2018 
17 - Antunes JLF, Narvai PC. Políticas de saúde bucal no Brasil e seu impacto sobre as desigualdades em saúde. Rev Saúde Pública, 2010; 44(2):360-5.

18 - Magno LD. Dos Clamores das Ruas aos Rumores no Congresso: uma análise da conjuntura recente da saúde no Brasil. [Dissertação]. Salvador: Instituto de Saúde Coletiva, Universidade Federal da Bahia, 2015.

19 - Cappellano MC. Nível de Informação do Usuário sobre Saúde Bucal em Unidades de Atenção Básica. [Dissertação]. Bauru: Universidade do Sagrado Coração, 2018.

20 - Franco DH. Financiamento Público e Privado da Saúde Bucal no Brasil e no Mundo. [Tese]. Piracicaba: Faculdade de Odontologia de Piracicaba da Universidade Estadual de Campinas, 2017. 